\title{
Transmission Sequence Design and Allocation for Wide-Area Ad Hoc Networks
}

\author{
Wing Shing Wong, Fellow, IEEE
}

\begin{abstract}
In this paper, we examine the problem of designing and allocating transmission sequences to users in a mobile ad hoc network that has no spatial boundary. A basic tenet of the transmission sequence approach for addressing media access control (MAC) is that under normal operating conditions, there is no feedback-triggered retransmission. This obviously is a major departure from the Slotted-ALOHA or carrier-sense multiple-access (CSMA)-type approaches. While these traditional solutions enjoy excellent throughput performance, a fundamental drawback is that they are based on feedback information. For systems without a naturally defined central controller that can play the role of a base station, the task of providing feedback information could easily become unmanageable. This highlights the advantage of the feedback-free approach. A second advantage is the ability to handle unlimited spatial coverage. We propose in this paper a concept for MAC that is akin to frequency reuse. However, instead of reusing frequency, the new approach allows transmission sequences to be reused. A study of the transmission sequence approach against other approaches is conducted by comparing the minimal frame lengths that can guarantee the existence of conflict-free transmissions.
\end{abstract}

Index Terms-Chinese remainder theorem (CRT) sequences, code-based scheduling, frequency reuse, Global Navigation Satellite Systems (GNSS), protocol sequences, user-irrepressible sequences.

\section{INTRODUCTION}

$\mathbf{O}$ $\mathrm{NE}$ of the basic challenges in wireless ad hoc network design is to ensure efficient simultaneous access to the shared communication media for massively many geographically distributed users. For wide-area multihop ad hoc networks, the issues could be even more demanding.

Traditional multiple-access controls are either frequencydivision based (FDMA), code-division based (CDMA), or timedivision based (TDMA). Standard FDMA or CDMA schemes contain elaborate steps to ensure that a transmitter and its intended receiver are synchronized to the same frequency band or the same spreading code. These steps are necessary because it is physically impossible to ask the receiver to monitor all transmission channels or the whole code space at the same time. Instead, the receiver and the transmitter must find a way to match each other. The matching algorithm usually requires some form of handshaking or acknowledgment message ex-

Manuscript received January 11, 2013; revised May 27, 2013; accepted August 4, 2013. Date of publication September 6, 2013; date of current version February 12, 2014. This work was supported by the National Natural Science Foundation of China under Grant 61174060 and Shenzhen matching Grant GJHS20120702105523301. The review of this paper was coordinated by Dr. J. Pan.

The author is with the Department of Information Engineering, The Chinese University of Hong Kong, Hong Kong (e-mail: wswong@ie.cuhk.edu.hk).

Digital Object Identifier 10.1109/TVT.2013.2280996 changes and is costly. For ad hoc networks supporting datagram services, these setup procedures may prove to be complicated and inefficient, thus motivating the search for protocols with simple setup requirements.

For the TDMA approach, a frequency channel is divided into concatenated time slots of fixed duration. These slots are usually grouped into periodic frames. While it is possible for a receiver to monitor all time slots, severe interference may occur if multiple users decide to broadcast at nearly the same time. Hence, some TDMA schemes also require slot allocation by a centralized controller, which will incur connection setup costs. Nevertheless, setup-free time-division-based schemes are also available, such as Slotted-ALOHA or carrier-sense multipleaccess (CSMA). These schemes are efficient but require channel information feedback for contention resolution. While feedback can be readily provided on systems with base stations, it poses cumbersome burdens for ad hoc networks that do not possess a differentiated control management hierarchy.

In this paper, we develop a setup-free approach based on two closely related concepts, namely, code-based scheduling and protocol sequence. Both of them are feedback-free. That is, under normal operating conditions, there is no need to feed the channel status back to the transmitters, thereby eliminating the requirement of a centralized controller. Both of these approaches define the access protocol by using binary transmission sequences to indicate permission to transmit or to remain silent for each time slot. Here, we follow the convention that a "one" represents permission to transmit and a "zero" to remain silent. The issue of defining an orderly and efficient access to the channel is then reduced to a question of designing transmission sequences that satisfy the following conditions.

1) System performance should achieve targeted objectives.

2) There should be a mechanism for users to acquire their transmission sequences automatically.

3) The sequence parameters should allow various traffic scenarios to be readily accommodated.

The statement for the first condition is deliberately vague since there can be a variety of system goals to be achieved, such as maximizing system throughput, minimizing average delay, minimizing maximum delay, and so on. In this paper, we focus on the objective of providing service guarantee that each user can broadcast at least one contention-free packet to its neighbors in each frame and that the frame length is minimized. However, our investigation can be extended to throughput maximization, for example.

No matter what the system objective is, the ideal solution would depend on the degree of time synchronization among users. Since Global Navigation Satellite Systems (GNSS) are 
ubiquitous these days and they can provide time synchronization, we assume that transceivers have access to a common GNSS and that their clocks are synchronized up to some intrinsic inaccuracies. However, due to propagation delay, it is not possible to perfectly synchronize the clocks among a transmitter and all of its receivers. Therefore, we need to discuss media access control (MAC) solutions for wide-area ad hoc networks under various synchronization conditions. Details will be described in subsequent sections.

For code-based scheduling, a simple approach for sequence distribution is to preassign them to all users (transceivers). This assumes that the maximum number of users in the network is unchanging and a priori known. While there are other alternatives for automatic transmission sequence allocation (see, for example, [1]), in this paper, we propose a scheme that is based on the geographic location of the distributed users. A key premise is that all users have access to their location information at marked time instances through the GNSS. To define the allocation scheme, we tessellate the geographic region to be covered into regular hexagonal quantization cells. The cell boundaries are assumed to be fixed and known to all. Therefore, all users can identify themselves with a location cell at any time instance marked by a GNSS signal broadcast. The transmission sequences are preassigned to the location cells and then dynamically assigned to the users depending on their location.

A distinct feature of our allocation scheme is that it takes advantage of the idea that users separated by distance larger than the hearing range can reuse the same time slots and, hence, can be assigned the same transmission sequence. This form of sequence reuse reminds us of, and yet is distinct from, frequency reuse in cellular networks.

Since the methodology of our solution approach comes from topology-transparent code-based scheduling and protocol sequence, it would be appropriate to recall basic ideas from these topics.

Starting from [2], various researchers have proposed using code-based scheduling to address the media access problem. The sequences defined in [3]-[5], for example, can be applied to the multihop ad hoc networks under consideration. By means of various coding schemes, such as Reed-Solomon (RS) codes, one can construct large sequence spaces to support the stated applications. However, a major issue is that these sequences require perfect time synchronization. In an ad hoc network, even if transmitters are perfectly synchronized to a global clock, the times when the packets are received are typically not, due to propagation delays.

Protocol sequences are more suitable for ad hoc network applications. In [6] and [7], Massey and his coauthors proposed using binary sequences to address the MAC problem for asynchronous users in a cell and coined the nomenclature. Simply put, protocol sequences are binary sequences with a common period and designed with special Hamming crosscorrelation properties. These sequences are expected to be used repeatedly in real applications. For asynchronous systems, the starting points of users are indeterminate; hence, cyclic shifts of a particular sequence should not be treated as distinct codes. Thus, protocol sequences are closely related to the cyclically permutable codes defined by Gilbert [8]. These are binary block codes with each codeword having a cyclic order that is equal to the block length. (The cyclic order of a code sequence is the minimal number of shifts required to bring it to its original form.) In [9], various methods for constructing cyclically permutable codes were proposed. These constructions were based on a classical application of the Chinese remainder theorem (CRT), which defines a one-to-one onto correspondence between vectors and matrices. Subsequently, the authors also used the CRT correspondence to construct new classes of protocol sequences that are known as CRT sequences (see [10]-[12]). These CRT sequences are closely related to the Generalized Prime Sequences defined in [1], which, in turn, are generalizations of prime sequences [13] and extended prime sequences [14]. We will show that cyclically permutable codes are excellent candidates for MAC application in ad hoc networks.

In the following sections, we describe how to design transmission sequences for wide-area ad hoc networks under various synchronization conditions. The GNSS-based solution is presented. This approach is based on transmission sequence reuse, which is a new concept modeled after frequency reuse. We also discuss the benefits of GNSS-based allocation. Among them is the ability to allow the maximum number of local users to vary according to location. We propose new sequence constructions to achieve this feature. The construction is based on the concept of product sequences, which also relies on the CRT correspondence.

The organization of the rest of this paper is as follows: The basic model and key assumptions are presented in Section II. In Section III, we recall relevant results and concepts from protocol sequences. Section IV describes a new sequence-allocation algorithm, i.e., the GNSS-based allocation, that relies on the geographical location of the users. In Section V, we present different transmission sequence sets that can be used in a GNSSbased allocation, including TDMA, code-based scheduling, and protocol sequences. In Section VI, we discuss the difference between the GNSS-based code allocation with traditional codebased scheduling. We also describe a new code allocation scheme that can adapt to geographically changing user density. Some concluding remarks are offered in Section VII.

\section{Multihop Ad Hoc Network}

A mobile multihop ad hoc network is a network without hierarchical infrastructure in which mobile users can broadcast data bursts to all their neighbors who are within hearing range [15]. Such type of models is appropriate for describing networks for vehicular applications (see, for example, [1] and [16]). It is difficult to achieve efficient MAC design using feedbacktype protocols for these networks since they contain massively many access points that render standard contention resolution algorithms cumbersome. Thus, feedback-free approaches are investigated in this paper.

Consider a multihop ad hoc network covering a geographic area without bounds; hence, it can support in theory an unbounded number of users. All users are mobile, with maximum mobility speed given by $v \mathrm{~m} / \mathrm{s}$. All users are continually active in the sense that they are always ready to transmit and to receive messages. Such a traffic data model is suitable for applications 
such as neighboring vehicle detection or collision warning in a vehicular ad hoc network (VANET).

All users share a single slotted frequency channel for halfduplex communication. That is, the channel is divided into time slots, and at each time slot, a mobile user can either transmit or receive signal but cannot do both. The time slots are all $\tau$ s long. A user coordinates transmission and reception in each frame by means of an assigned transmission sequence that has a period equal to $L$. This number also defines the number of slots in a frame. Unlike code-based scheduling approaches, which allocate sequences to users directly, we consider an alternative approach in which sequences are first allocated to geographic cells and then to the users through their cell location, as explained in Section IV.

We assume that all users have access to a GNSS that broadcasts signals at marked time instances, i.e., $\left(S_{0}, S_{1}, S_{2}, \ldots\right)$, where $S_{i}=i T$ for some $T$. The time between any two consecutive marked instances is organized into a superframe consisting of $F$ frames, each of which contains $L$ time slots of length $\tau$. Therefore

$$
T=F L \tau+T_{G}
$$

where $T_{G}$ stands for the superframe guard time that is added to ensure all packets transmitted in a superframe will be received during the same superframe. An upper bound for $T_{G}$ will be shown later. We refer to all frames other than the first and last one as normal frames. Our analysis will focus on normal frames to avoid complexities due to boundary efforts. For large values of $F$, the boundary effects are negligible.

As common in the analysis of ad hoc networks, we adopt the following assumption.

Interference Assumption: Any receiver can only listen to those transmitters within a distance $R$ from it, transmissions from location at a distance equal to or greater than $R$ are simply ignored. Since we are mainly interested in scenarios where the value of $T$ is in the order of seconds, we assume that the interference relations among users are unchanged during a superframe, i.e., $\left[S_{i}, S_{i+1}\right)$, to simplify the analysis.

For proper protocol operation, users are required to synchronize to the global GNSS clock by aligning to the marked instances-the exact start time of the $k$ superframe is announced by the GNSS at $S_{k}$. However, due to differences in propagation delay and processing time, actual start times for different users may differ. If $t_{i}$ represents the start time of a superframe for user $i$, we assume that there is a system-wide integer bound, i.e., $\Delta_{C}$, such that

$$
0 \leq t_{i}-S_{i} \leq \tau \Delta_{C}
$$

Let

$$
\Delta_{P}=\left\lceil\frac{R}{c \tau}\right\rceil
$$

where $c$ is the speed of light. Define

$$
\Delta=\Delta_{C}+\Delta_{P} .
$$

We assume that

$$
\Delta \leq L
$$

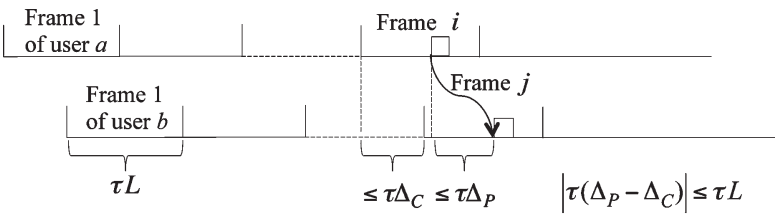

Scenario 1: Superframe of user $a$ starts first.

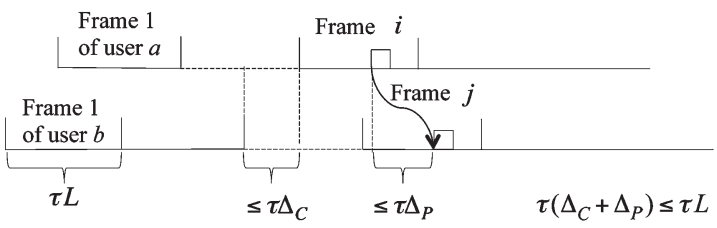

Scenario 2: Superframe of user $b$ starts first.

Fig. 1. Two scenarios for calculating time differences between users.

It follows that if user $a$ transmits a packet at a slot in a normal frame $i$ and the packet is received by user $b$ during frame $j$, then the difference between $i$ and $j$ is bounded by 1 . That is

$$
|i-j| \leq 1 \text {. }
$$

See Fig. 1 for illustration.

It also follows that the superframe guard time can be set to $\tau \Delta$.

In our analysis, we assume different types of synchronization, which are defined in terms of the value of $\Delta$ and $\Delta_{C}$.

1) The system is perfectly clock synchronized if $\Delta_{C}=0$.

2) The system is perfectly synchronized if $\Delta=0$.

3) The system is partially synchronized to $\Delta_{0}$ slots if $\Delta=$ $\Delta_{0}<L$; the system is asynchronous if $\Delta=L$.

It is worthwhile to point out that although these definitions are expressed in terms of a multiple number of slots, the time differences can be any positive real value bounded by $\tau \Delta$. In subsequent discussions, we also need to refer to the concept of slot synchronized; this refers to cases where the time difference for any arbitrary transmitter-receiver pair is idealized to an integral multiple of $\tau$ for discussion simplicity.

Inaccuracies in information are not restricted to time and may also occur in the location coordinates of the users. These inaccuracies are taken care of by means of quantization of location coordinates into regular hexagonal tiling cells with cell radius $h \mathrm{~m}$. To be more precise, consider a planar coordinate system with the axes intersecting at a $60^{\circ}$ angle instead of orthogonally. We can define a regular tiling of the plane by hexagonal cells with centers $(m d, n d)$ for all integers $m$ and $n$, where $d=\sqrt{3} h$, as shown in Fig. 2. We call these cells quantization cells.

A fundamental assumption of our model is as follows.

Spatial Coordinate Quantization Assumption: There is a well-defined mapping taking the GNSS location information into quantization cells. Any user can determine, at the local start time of a superframe, the identity of the cell to which it belongs.

Although there is no upper bound on the number of users in the network, there is a bound on the number of users within a hearing distance area. We refer to this bound as the maximum number of local users. In particular, we adopt the following. 


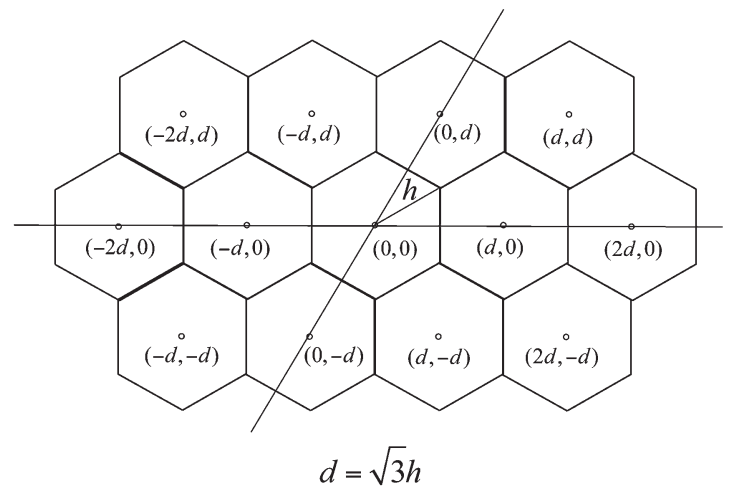

Fig. 2. Hexagonal quantization of the transceiver coordinates.

Maximum Interferer Assumption: At any time and at any location, there is no more than $M$ users in any area of size $\pi R^{2}$. Hence, the maximum number of interferers is $M-1$.

We are now ready to define the problem to be addressed in this paper.

Definition 2.1: Consider a multihop ad hoc network that satisfies all the assumptions stated in this section. The network is said to provide block-free service with parameters $(M, L)$ if it employs transmission sequences with period $L$ and that when the maximum number of local users everywhere is not more than $M$, any user in the network can receive at least one contention-free transmission in each normal frame of a superframe from any user who is located within its hearing distance at the beginning of that superframe. (Note that by the Interference Assumption, the interference relations among users are unchanged within a superframe; therefore, users within hearing range will remain so in the whole superframe.)

If a network can provide block-free service with parameters $(M, L)$, it can also guarantee that any user can transmit at least one contention-free packet in each of normal frame of a superframe to any user who is located within its hearing distance when that superframe starts.

This type of service guarantee is appropriate for applications in which total system throughput is not a primary concern, but instead, the focus is on how to guarantee all users can connect to their neighbors within time constraints. That is, it is important to ensure that all users can transmit to and hear from its neighbors within a bounded time delay. Such a guarantee cannot be provided under probabilistic protocols, such as CSMA.

In subsequent sections, we examine the question of how to design and allocate transmission sequences under different synchronization assumptions. One basic objective is to find sequences with the shortest sequence length. We will only focus on solutions that do not require information on current transmission status; hence, algorithms that require users to monitor the channel before transmission are excluded. Adding such a step could enhance performance, although at extra cost.

\section{Relevant Concepts From Protocol Sequences}

We review in this section coding theory and protocol sequence results that would be needed in subsequent sections. First of all, we specify the idea of a protocol sequence set.
Definition 3.1: A protocol sequence set $\mathcal{P}$ consists of binary sequences having a common period $n$. The weight of an element $X \in \mathcal{P}$ is the number of 1's in it and is denoted by $w(X)$.

Define the rightward cyclic shift operation, i.e., $\mathcal{R}$, on sequence $X$ by

$$
\mathcal{R}(X(1), X(2), \ldots, X(n))=(X(n), X(1), \ldots, X(n-1))
$$

where $X(i)$ denotes the $i$ th component of $X$. By a slight abuse of notation, the same $\mathcal{R}$ represents the rightward cyclic shift operation on sequences with different lengths. The Hamming cross-correlation function of elements $X$ and $Y$ can be expressed as

$$
H(X, Y)(\tau)=\sum_{i=0}^{n-1} X(i) \mathcal{R}^{\tau} Y(i)
$$

A cyclically permutable code is defined to be a binary block code with length $n$ such that all codewords have $n$ distinct cyclic shifts, and all codewords are cyclically distinct. For such codes, it is useful to extend the minimum Hamming distance to cyclic minimum distance, i.e., $d_{c}$, which is the minimum Hamming distance between all cyclically shifted versions of any two codewords. That is

$$
d_{c}=\min _{X, Y} \min _{i} d\left(X, \mathcal{R}^{i} Y\right)
$$

where $d$ is the regular distance function between two codewords.

In [9], several methods for constructing cyclically permutable codes were proposed. One of the approaches, which is based on RS codes, is relevant to our discussion. Let $q$ be a prime number. Consider an RS code, i.e., $C(n, q, k)$, which has codewords of length $n$ over $\operatorname{GF}(q)$. For $0 \leq i<q$, let $E_{i}(q)$ represent the sequence

$$
E_{i}(q)=(\overbrace{\underbrace{0, \ldots, 0}_{i}, 1,0, \ldots, 0)}^{q} .
$$

Denote the set $\left\{E_{1}(q), \ldots, E_{q}(q)\right\}$ by $\mathcal{E}(q)$. The $v_{q^{-}}$ representation is a mapping from $\mathrm{GF}(q)$ to $\mathcal{E}(q)$ that maps $j$ to $E_{j}(q)$. A codeword from $C(n, q, k)$ can then be viewed as a $q \times n$ binary matrix. Moreover, it is shown in [9] that this construction defines a cyclically permutable code with codeword length $n q$, constant weight $n$, codeword size $q^{(k-2)}$, and minimum distance $2(n-k+1)$, for $3 \leq k<n<q$.

In the given result, the connection going from a $q \times n$ matrix to a length $n q$ sequence is based on the CRT correspondence (denoted by $\gamma$ ), which is a mapping taking entries of a $c d$ dimensional vector to entries of a $c \times d$ matrix. If $c$ and $d$ are relatively prime, the CRT implies that such a correspondence is one-to-one and onto. Moreover, it is proved in [9] that a cyclic rightward shift of a $c d$-dimensional vector is equivalent to a cyclic downward row shift followed by a cyclic rightward column shift of the corresponding matrix. 
The CRT correspondence has also been employed to construct several classes of protocol sequences, which are known as CRT sequences. There are several variants of CRT sequences. We recall the definition of a class of CRT sequences and their basic properties from [11] to facilitate subsequent discussions.

Definition 3.2: Let $p$ and $q \geq 2 p-1$ be relatively prime positive integers. A CRT sequence set, i.e., $\mathcal{C}(p, q)$, consists of $p$ binary sequences with period $p q$ such that each element in it has a characteristic set (the set of positions in the sequence with value 1) that can be summarized as

$$
\begin{aligned}
\mathcal{I}_{g}=\{l \in \mathbb{Z}: 0 \leq l<p q, \gamma(l)= & (j g \bmod p, j) \\
& j=0,1, \ldots, p-1\}
\end{aligned}
$$

for $0 \leq g<p$. Parameter $g$ is referred to as the sequence generator.

Each of the $p$ distinct sequences in the set has a Hamming weight $p$. Moreover, the Hamming cross-correlation between any shifted versions of any two distinct sequences is either 0 or 1 , which implies that the sequence set possesses the user irrepressible (UI) property. This terminology was initiated in [17] and [18] and was given the following mathematical definition in [19].

Definition 3.3: Consider a protocol sequence set with $k$ elements, each having a period $l$. Each element is represented by a shifted version that is obtained by applying the operator $\mathcal{R}$ independently for an arbitrary number of times. Denote by $\mathbf{M}$ the $k \times l$ matrix obtained by stacking these representations one above the other. The protocol sequence set is UI if we can always find a $k \times k$ submatrix of $\mathbf{M}$, which is a permutation matrix, regardless of the number of shifts applied to produce the representations.

The relevancy of UI to a slot-synchronized network can be apprehended by interpreting $k$ as the number of users and the protocol sequences as transmission sequences. The UI condition is equivalent to the requirement that no matter what the relative shifts are, each user is guaranteed to have one conflictfree transmission in each period of $l$ slots if propagation delays are ignored. We use the phrase "a conflict-free 1" to refer to a sequence entry that corresponds to a column with only one nonzero entry in $\mathbf{M}$.

The CRT sequence sets are among the shortest known UI sequence sets. In [11], there is a table summarizing the shortest UI sequence sets for small user numbers. In it, many of the known cases are CRT-type sequences.

We conclude this section with a slightly modified version of a CRT sequence set for our subsequent discussion.

Definition 3.4: Let $p$ and $q \geq 2 p-1$ be relatively prime positive integers. A $\mathcal{C}_{0}(p, q)$ sequence set consists of $p$ binary sequences with period $p q$ of the following types.

1) For $g$ in $\{0\} \cup\{2, \ldots, p\}$, there are $p-1$ CRT sequences, i.e., $S_{g}$, with characteristic set $\mathcal{I}_{g}$.

2) A single sequence, i.e., $S_{*}$, with characteristic set

$\mathcal{I}_{*}=\{l \in \mathbb{Z}: 0 \leq l<p q, \gamma(l)=(j, 0), j=0,1, \ldots, p-1\}$.
It follows from results in [11] that the following holds.

Lemma 3.1: If $q \geq 2 p-1$, the Hamming cross-correlation function of any two distinct sequences in $\mathcal{C}_{0}(p, q)$ is upper bounded by 1 .

\section{Basics of Global Navigation Satellite System-BAsed Code Allocation}

Here, we describe how transmission sequences are distributed to the users, assuming a sequence set has already been specified. In traditional code-based scheduling models, transmission sequences are preassigned to users. For applications such as VANETs, since all cars in a country may be potential users, the dimension of the required code space could be extreme large, although it is unlikely that any two users would interfere with each other. In fact, in this paper, we assume the number of users to be theoretically unbounded. However, the number of users within a geographical region is bounded and likely to be small. Hence, we propose an alternative sequence distribution scheme that is based on the instantaneous geographical location of the users. Here, we describe in detail how users can acquire transmission sequences automatically based on their geographical locations.

Recall that under our stated assumptions, all users acquire a unique cell identity number at the start time of their local superframes. The transmission sequence will be based on this cell identity. To avoid duplications in the sequence assignment, it is necessary that no two users will acquire the same cell identity at the same superframe. (The perceived start times of the superframes may differ as explained in Section II.)

If all users are perfectly clock synchronized, this requirement translates to the condition that no two users can occupy the same quantization cell at the same time. Since the quantization cells are small, this is a mild condition to impose. For the general case where users are not perfectly synchronized, let $\tau_{i}(k)$ represent the start time of superframe $k$ for user $i$, and let $\left(x_{i}(k), y_{i}(k)\right)$ represent the quantization cell at which the user is located at: $\tau_{i}(k)$. To maintain unique code sequence assignment, we require that the following condition holds.

Fermion Condition: For any superframe $k$, if $\left(x_{i}(k)\right.$, $\left.y_{i}(k)\right)=\left(x_{j}(k), y_{j}(k)\right)$, then $i=j$.

If $\tau_{i}(k)=\tau_{j}(k)$, the Fermion condition just requires that the users cannot occupy the same quantization cell at the same time. Since $\tau_{i}(k)$ and $\tau_{j}(k)$ can be different, this condition requires that the users need to be further apart than in the synchronized cases so that the maximum distance traveled during the time gap cannot affect the uniqueness in quantization. For example, consider a case where $h=0.5 \mathrm{~m}, \Delta \tau=100 \mathrm{~ms}$, and $v=$ $60 \mathrm{~km} / \mathrm{hr}$; then, as long as the users are not closer than $4.34 \mathrm{~m}$ at any time, the Fermion condition will hold. For VANET applications, this is not a severe restriction.

The transmission sequence allocation is defined by a mapping, i.e., $\alpha$, from $\mathcal{C}$, the set of cell identities, to $\mathcal{L}$, the set of defined transmission sequences. It is assumed that this mapping is downloaded to all users at the time when they join the network and can be updated from time to time, but not dynamically. Since $\mathcal{C}$ is theoretically an unbounded set and $\mathcal{L}$ is finite, it is necessary to reuse some of the sequences in $\mathcal{L}$. 
As in the solution to frequency channel allocation, we address this issue by defining a basic tiling pattern, in other words, a cell cluster, and impose the condition that $\alpha$ maps cells in the cluster to distinct sequences. The tiling pattern is then repeated to cover the whole area and thereby extending $\alpha$ to all of $\mathcal{C}$. It follows that $\alpha$ can be easily computed with a finite amount of data storage.

Under the Interference Assumption, users can hear each other only if their distance separation is less than $R$ at the beginning of a superframe. However, due to the hidden node problem, a receiver may hear interfering signals from multiple transmitters separated by a distance less than $2 R$.

As a result, it is permissible to share the same transmission sequence only for users separated by a distance greater than or equal to $2 R$. Since the quantization cells are small compared with $R$, there is little error in assuming that the users are always located at the cell centers. This assumption enables us to simplify the allocation constraint as follows.

Sequence Allocation Constraint: Cells separated by a distance less than $2 R$ cannot share the same transmission sequence.

Allocation algorithms satisfying this condition have already been invented in the context of FDMA. The tiling pattern can be determined to satisfy this constraint according to the algorithm in [20]. For completeness, we will briefly summarize the procedure here. Let $G$ be the smallest integer that satisfies the following condition:

$$
b_{1}^{2}+b_{1} b_{2}+b_{2}^{2}=G \geq\left(\frac{2 R}{d}\right)^{2}
$$

for some nonnegative integers, i.e., $b_{1}$ and $b_{2}$.

If a cell centered at $\left(m_{1}, m_{2}\right) d$ is assigned a sequence $S_{1}$, we assign the same sequence to all cells centered at

$$
\left(m_{1}+i b_{1}, m_{2}+j b_{2}\right) d
$$

for arbitrary integers $i$ and $j$. If there is another cell centered at a distance less than $2 R$ from $\left(m_{1}, m_{2}\right) d$, a different sequence other than $S_{1}$, for example, $S_{2}$, should be assigned. Then, $S_{2}$ can be assigned to a lattice of cells in a way similar to $S_{1}$. This allocation process is repeated until all cells are assigned a sequence.

It is proven in [20] that the minimal number of sequences required to fill the whole space so that no cells with distance $2 R$ are assigned the same sequence is equal to $G$. For example, consider a case where the quantization radius is $h=1 \mathrm{~m}$ and $R=0.5 \mathrm{~km}$; then, $G=333,333$. This is not a small number, but it could be a couple of orders less than the total number of users in a system. For example, the number of registered vehicles in a country could be on the order of several tens of millions.

In subsequent sections, we will consider how to construct enough sequences so that all users can be guaranteed the UI property. We also want to achieve this with a short frame period. While there are different methods for constructing UI sequences, a common theme is that the mapping taking a cell in a cluster to a sequence can be arbitrarily defined as long as it satisfies the one-to-one condition. This approach greatly simplifies the transmission sequence design.

\section{Performance of Global Navigation Satellite SYSTEM-BASED SOLUTIONS}

Before discussing solutions based on protocol sequences, we present several baseline models for comparison. The first is TDMA scheduling.

Assume that the system is perfectly synchronized, that is, $\Delta=0$. For TDMA scheduling, we assign a slot to each cell in a cell cluster. Thus, the minimum frame size is $G$, the value of which is defined in (4.1). Hence

$$
L=G \text {. }
$$

When propagation delay and clock differences are taken into account, that is, when $\Delta \neq 0$, there is no simple way to guarantee UI property to all users except by appending to every time slot $\Delta$ silent slots in which no transmission is allowed. These extra time slots allow propagation delay and clock difference effects to be isolated from one transmission to the next. Hence, the minimum frame size is

$$
L=(\Delta+1) G .
$$

To appreciate the performance of this approach, consider an example where $h=1 \mathrm{~m}, R=0.5 \mathrm{~km}$, and $\Delta=10$. According to (4.1), this implies that the minimum period of a TDMA time frame in which each user can be guaranteed to enjoy at least one data burst conflict-free is 3333333 . In most applications, it is unlikely that the number of users within a radius of half a kilometer would exceed tens of thousands. Thus, the TDMA scheme is extremely inefficient for this scenario. Generally speaking, unless $M$ is on the order of $G$, TDMA is not an efficient choice.

For a sequence set with constant weight $w$, to achieve the UI property, it is necessary that

$$
\begin{aligned}
& w \geq M \\
& L \geq w .
\end{aligned}
$$

These bounds are optimistic. It is shown in [11] that for slotsynchronized cases, given any UI protocol sequence set that supports $M$ users, the sequence length, i.e., $L(M)$, must satisfy

$$
L(M) \geq\left\lceil\frac{8 M^{2}}{9}\right\rceil .
$$

That is, the lower bound is quadratic in $M$. Below, we identify two sequence sets that satisfy the quadratic growth rate.

For the perfectly synchronized cases, there is a host of solutions in the literature under the heading of code-base scheduling, topology-transparent scheduling, or time-spread multiple access (see, for example, [3]-[5] and [21]-[23]). As previously explained, these approaches allocate the transmission sequences directly to the users rather than to the cells. If $N$ denotes the number of users in the network, then it is shown in [3] that one can construct a sequence set with period $L$ satisfying the order condition, i.e.,

$$
L=O\left(\frac{M^{2} \ln ^{2} N}{\ln ^{2} M}\right) .
$$


In [4], a solution based on a $C(n, q, k)$ RS code has been proposed, which has the same order property.

If we adopt these sequences to a GNSS-based system and assign them to cells in a cluster, the network can provide blockfree service with appropriate parameters.

Proposition 5.1: For a network under partial synchronization up to $\Delta$, a code-based scheduling can provide block-free service with parameters $(M, L)$, where

$$
L=O\left((\Delta+1) \frac{M^{2} \ln ^{2} G}{\ln ^{2} M}\right)
$$

where $G$ is the number of cells in a cell cluster.

Proof: Consider an arbitrary user, i.e., $u$, located at a quantization cell with center $O$ at time $S_{k}$. Users who are located in cells with centers at a distance less than $R$ from $O$ at time $S_{k}$ are the only users who can be heard by $u$ during the superframe according to the Interference Assumption. By the Maximum Interferer Assumption, the total number of such users including $u$ is at most $M$. There exists a $C(n, q, k) \mathrm{RS}$ code with codeword size $q^{k}$ satisfying

$$
\begin{aligned}
q^{k} & \geq G \\
n & \geq(k-1)(M-1)+1 \\
q & \geq M .
\end{aligned}
$$

Moreover, the period of the corresponding transmission sequences is $n q$. By the construction of the cell cluster, all these $M$ users will be assigned distinct transmission sequences, since the distance between any two of them is less than $2 R$. If $\Delta=0$, these conditions imply that all users can transmit at least one conflict-free packet during a frame of $n q$ slots. This implies that $u$ can receive at least one packet from each of the $M-1$ users during a normal frame. Following the same argument in [3], it is easy to show that

$$
n q=O\left(\frac{M^{2} \ln ^{2} G}{\ln ^{2} M}\right) .
$$

For the partially synchronized case, we can maintain the UI property if we append every time slot in the RS code sequence by $\Delta$ silent slots in which no transmission is allowed. So that

$$
L=(\Delta+1) n q .
$$

Even when $\Delta$ is of moderate value, the code-based scheduling approach could be impractical. However, one can adopt shorter solutions based on protocol sequences or cyclically permutable codes. As mentioned in Section III, there exists cyclic permutable codes with length $n q$, constant weight $n$, codeword size $q^{k-2}$, and cyclic minimum distance $d_{c} \geq 2(n-k+1)$ for $3 \leq k<n<q$. We can use this code to provide a solution to the asynchronous case by assigning distinct codewords to each cell within a tiling pattern. Hence

$$
\begin{aligned}
& q^{k-2} \geq G \\
& n \geq(k-1)(M-1)+1 \\
& q \geq M .
\end{aligned}
$$

Proposition 5.2: For the asynchronous case, it is possible to provide $(M, L)$ block-free service using cyclically permutable codes with the period satisfying

$$
L=O\left(\frac{M^{2} \ln ^{2} G}{\ln ^{2} M}\right) .
$$

Proof: The proof for the slot-synchronized case is similar to the proof of Proposition 5.1. The cyclically permutable property implies that the UI property is maintained, regardless of the relative shifts of the sequences. For the asynchronous case, the transmission of a user may lie in between two consecutive slots of the receiver. However, as suggested in [6], if we append to every slot of the code sequence an extra silent slot in which no transmission is allowed, the UI property will be preserved. It follows that

$$
L=2 n q
$$

Using arguments similar to [3], the estimate for $L$ follows.

\section{Location-Based Protocol Sequence Design}

Comparing the GNSS-based allocation with the traditional code-based scheduling, an obvious difference is that GNSSbased allocation sets no a priori limit on the number of users in the system. Another important difference is that GNSSbased allocation offers the possibility for the allocation to be dependent on the geographic location. For example, user density clearly depends heavily on location; the number of users per unit area is higher in downtown districts than in the countryside. Allocation schemes that are designed based on the maximum number of interferers seen in a downtown district may prove to be too pessimistic in the countryside.

The protocol sequence sets we introduce so far are designed based on the assumption that the maximum number of interferers, i.e., $M-1$, is constant in the whole geographic area. Here, we consider the issue of designing protocol sequence sets that allow variation on the maximum of number of interferers while maintaining the UI property. Our proposed solution is based on the idea of sharing a protocol sequence among multiple users. The sharing is enabled by means of product sequences. These objects are distinct from product codes, in spite of some superficial similarities (see [24] for a definition of product codes).

Consider $\mathcal{P}$ and $\mathcal{Q}$, i.e., two protocol sequence sets with sequence length $p$ and $q$, respectively. Let $X \in \mathcal{P}$ and $Y \in \mathcal{Q}$.

Definition 6.1: Suppose $p$ and $q$ are relatively prime, the product sequence of $X$ and $Y$, denoted by $X \otimes Y$, is defined as follows. Let $\mathbf{M}_{X, Y}$ be the $p \times q$ matrix with the $(i, j)$ entry defined by

$$
\mathbf{M}_{X, Y}(i, j)=X(i) Y(j) .
$$

$X \otimes Y$ is the unique binary sequence that corresponds to $\mathbf{M}_{X, Y}$ via the CRT correspondence.

It is clear that the length of $X \otimes Y$ is $p q$, and it has weight equal to $w(X) w(Y)$. 
Lemma 6.1: The following properties hold for product sequences.

1) If $X \otimes Y=X^{\prime} \otimes Y^{\prime}$ is not the all-zero sequence, then

$$
X=X^{\prime}, \quad Y=Y^{\prime} .
$$

2) $\mathcal{R}(X \otimes Y)=\mathcal{R}(X) \otimes \mathcal{R}(Y)$.

The proofs of these results are straightforward and are omitted.

Consider a cyclically permutable protocol sequence set, i.e., $\mathcal{P}$, such as the one constructed in Section V. Recall that we can construct it based on a $C(n, q, k) \mathrm{RS}$ code so that the cyclic minimum distance satisfies $d_{c} \geq 2(n-k+1)$. For simplicity, we assume that $n$ and $q$ are primes. Moreover

$$
n \geq(k-1)(M-1)+1 .
$$

It follows that any subset of $\mathcal{P}$ with $M$ sequences is UI. We now show how to expand $\mathcal{P}$ to a larger protocol sequence set, i.e., $\widetilde{\mathcal{P}}$, to accommodate more users while maintaining the UI property. Let

$$
L=n q
$$

represent the sequence length of elements in $\mathcal{P}$. Let $p$ be a prime number such that $p \leq M$. Let $\mathcal{Q}=\left\{S_{1}, \ldots, S_{p}\right\}$ be a subset of $\mathcal{P}$ and $\mathcal{Q}^{C}$ be its complement in $\mathcal{P}$.

The basic idea of the construction is that sequences in $\mathcal{Q}$ will be split to support additional users, whereas sequences in $\mathcal{Q}^{C}$ are unchanged. Regions using split codes can allow for more interfering users without affecting nearby regions where the original codes from $\mathcal{Q}^{C}$ are used.

In mathematical notation, this can be formalized as follows. Let $U$ represent the all " 1 " sequence, i.e.,

$$
(\underbrace{1,1, \ldots, 1}_{p(2 p-1)}) \text {. }
$$

For any sequence $X$ in $\mathcal{Q}^{C}$, the product sequence $U \otimes X$ repeats $p(2 p-1)$ times the sequence $X$, and hence, its application essentially has the same effect as $X$ if the superframe is long enough. Let

$$
\widetilde{P}_{1}=\left\{U \otimes X: X \in \mathcal{Q}^{C}\right\} .
$$

Label elements in the CRT sequence set, i.e., $\mathcal{C}_{0}(p, 2 p-1)$, by $\left\{C_{1}, \ldots, C_{p}\right\}$. Define the set $\widetilde{P}_{2}$ as follows:

$$
\widetilde{P}_{2}=\left\{C_{i} \otimes S_{i}: 1 \leq i \leq p\right\} .
$$

Strictly speaking, the set $\widetilde{P}_{2}$ depends on how elements in the corresponding sets are labeled. However, for the results presented below, the ordering bears no important consequence, and any ordering could be used. The expanded set, i.e., $\widetilde{\mathcal{P}}$, is defined to be the union of $\widetilde{P}_{1}$ and $\widetilde{P}_{2}$. Note that sequences in $\widetilde{\mathcal{P}}$ have a sequence length equal to $p(2 p-1) n q$. Those belonging to $\widetilde{P}_{1}$ have weight $p(2 p-1) n$, and those belonging to $\widetilde{P}_{2}$ have weight $p n$.
Theorem 6.1: Any subset of $\widetilde{P}$ that includes all elements of $\widetilde{P}_{2}$ and $M-1$ elements from $\widetilde{P}_{1}$ is UI. Moreover, each sequence from $\widetilde{P}_{1}$ has at least

$$
p(2 p-1)-p(p-1) / 2=p(3 p-1) / 2
$$

conflict-free 1's in each period.

Proof: Let $S=C_{i} \otimes S_{i}$ be a protocol sequence from $\widetilde{P}_{2}$. Since $C_{i}$ is an element from the UI protocol sequence set, i.e., $C_{0}(p, 2 p-1)$, it has at least one conflict-free " 1 " no matter how the sequences in $C_{0}(p, 2 p-1)$ are shifted by $\mathcal{R}$. This means that in the matrix representation, $S$ has one row that does not conflict with any other sequences from $\widetilde{P}_{2}$. Since there are, at most, $M-1$ elements from $\widetilde{P}_{1}$, there is at least one conflictfree " 1 " entry in that row for $S$.

Now, suppose the subset contains a sequence $S$ that belongs to $\widetilde{P}_{1}$. Let $\mathbf{M}(S)$ be the matrix representation of $S$ and consider an arbitrary row, for example, row $r$ of $\mathbf{M}(S)$. There are, at most, $M-2$ other elements from $\widetilde{P}_{1}$ in the sequence subset. Hence, at most $(M-2)(k-1)$ of the 1's in row $r$ of $S$ conflict with those coming from sequences that belong to $\widetilde{P}_{1}$. Therefore, the number of " 1 " entries of $S$ in row $r$ that do not conflict with 1's from other sequences belonging to $\widetilde{P}_{1}$ is at least

$$
n-(M-2)(k-1) \geq k \text {. }
$$

Since any two sequences in $\mathcal{Q}^{C}$ can have, at most, $k-1$ bits in conflict, $S$ will have at least one conflict-free entry in row $r$ if there is no more than one sequence from $\widetilde{P}_{2}$ that has nonzero entries in row $r$ in its matrix representation. However, as $C_{0}(p, 2 p-1)$ is a UI protocol sequence set, there can be no more than $p(p-1) / 2$ places where elements from $C_{0}(p, 2 p-1)$ conflict with one another. Hence, $S$ has at least $p(2 p-1)-p(p-1) / 2$ conflict-free "1" entries.

Theorem 6.1 implies that, on the average, the effect on the nonsplit sequences is not too severe if one sequence is shared among up to $M$ users. By further analysis, we can show that for the nonsplit sequences, the delay between conflict-free " 1 " entries is bounded by

$2 p L$.

To prove this result, we need to establish the following.

Lemma 6.2: For any sequence $S$ in $C_{0}(p, 2 p-1)$, the minimal separation between two consecutive 1's is at least $p$. That is, there are at least $p-10$ 's between any two 1's.

Proof: It is easy to check that the claim holds for $S_{0}$ and $S_{*}$. Consider $S_{g}$ with $2 \leq g<p$. Recall that the characteristic set of $S_{g}$ is

$$
\begin{array}{r}
\mathcal{I}_{g}=\{l \in \mathbb{Z}: 0 \leq l<p(2 p-1), \gamma(l)=(j g \bmod p, j) \\
j=0,1, \ldots, p-1\} .
\end{array}
$$

Let $l^{*}$ be the unique solution satisfying the following equations. (Recall that $p$ is a prime.)

$$
\begin{cases}l \equiv 1 & \bmod 2 p-1 \\ l \equiv g & \bmod p \\ 0 \leq l<p q . & \end{cases}
$$


Then, $\mathcal{I}_{g}$ can be rewritten as

$$
\left\{0, l^{*}, 2 l^{*} \bmod p(2 p-1), \ldots,(p-1) l^{*} \bmod p(2 p-1)\right\} .
$$

We can relabel this set as $\left\{0, a_{1}, \ldots, a_{p-1}\right\}$ with the additional condition that

$$
0<a_{1}<\cdots<a_{p-1}
$$

One can show by induction that the distance between any two entries in the characteristic set of $S_{g}$ is not less than the distance between $a_{1}$ and 0 as well as $a_{p-1}$ and $p(2 p-1)$. That is, the minimal distance is equal to

$$
\min \left(a_{1}, p(2 p-1)-a_{p-1}\right) .
$$

For $g \neq 1$ and $l<p$

$$
l g \neq l \bmod p \text {. }
$$

Hence, for $j=0,1, \ldots, p-1$, there exists no solution to the following equation:

$$
(l, l)=(j g \bmod p, j)
$$

with $l$ in the range $1, \ldots, p-1$. On the other hand, for $l$ in this range, $\gamma(l)=(l, l)$, and therefore

$$
\gamma(l) \neq(j g \bmod p, j) .
$$

In other words, $l=1, \ldots, p-1$ cannot appear in $\mathcal{I}_{g}$. Similarly, for $l=p(2 p-2)+1, \ldots, p(2 p-1)-1$

$$
\gamma(l)=(l-p(2 p-2), l-(p-1)(2 p-1)) .
$$

Since

$$
l-(p-1)(2 p-1) \geq p(2 p-2)+1-(p-1)(2 p-1)=p
$$

$l=p(2 p-2)+1, \ldots, p(2 p-1)-1$ cannot appear in $\mathcal{I}_{g}$. Therefore

$$
\min \left(a_{1}, p(2 p-1)-a_{p-1}\right)>p-1 .
$$

Lemma 6.3: Consider the protocol sequence set $C_{0}(p, 2 p-$ $1)$. Let $\mathbf{M}$ be a $p \times p(2 p-1)$ binary matrix obtained by listing the sequences in the set as rows. Let $\mathbf{F}$ be derived from $\mathbf{M}$ by applying the operator $\mathcal{R}$ to each row independently for an arbitrary number of times. In $\mathbf{F}$, there is at least one zero column within any $2 p$ consecutive columns.

Proof: Without loss of generality, assume that the first $2 p$ columns of the matrix contain no zero column. Since the separation between the " 1 " entries in each row is at least $p$, the " 1 " entries in the first $p$ columns must come from different sequences. Similarly, the same holds for the $p+1$ th to $2 p$ th columns. Hence, for each sequence in $C_{0}(p, 2 p-1)$, i.e., $S_{i}$, we can identify two numbers $\left(a_{i}, b_{i}\right)$, which indicate the positions of two of its " 1 " entries, such that

$$
\begin{aligned}
& 1 \leq a_{i} \leq p \\
& p+1 \leq b_{i} \leq 2 p \\
& a_{i} \neq a_{j}, \quad \text { if } i \neq j, \quad b_{i} \neq b_{j}, \quad \text { if } i \neq j .
\end{aligned}
$$

Therefore

$$
\sum_{i=1}^{p}\left(b_{i}-a_{i}\right)=\sum_{i=p+1}^{2 p} i-\sum_{i=1}^{p} i=p^{2} .
$$

On the other hand, Lemma 6.2 implies that

$$
b_{i}-a_{i} \geq p
$$

As $C_{0}(p, 2 p-1)$ is a UI set and since the difference between consecutive " 1 " entries in $S_{0}$ is exactly $p, b_{i}-a_{i}$ is larger than $p$ for the other sequences. Hence

$$
\sum_{i=1}^{p}\left(b_{i}-a_{i}\right)>p^{2}
$$

which is a contradiction.

Theorem 6.2: Consider a subset of $\widetilde{P}$ that includes all elements of $\widetilde{P}_{2}$ and $M-1$ elements from $\widetilde{P}_{1}$. Let each element of the subset be represented by a shifted version that is obtained by applying the operator $\mathcal{R}$ independently for an arbitrary number of times. Form a matrix by stacking these representations one above the other. For each row corresponding to a sequence in $\widetilde{P}_{1}$, the gap between consecutive conflict-free 1 's in the matrix is at most

$$
2 p L \text {. }
$$

Proof: Let $S=U \otimes X$ be a sequence from $\widetilde{P}_{1}$. Without loss of generality, we can assume in the matrix representation of $S$ that the entry at the first row and first column corresponds to a conflict-free " 1 " and that for all sequences from $\widetilde{P}_{2}$, the first row contains only value 0 . Let $k$ be the nearest row in which all sequences from $\widetilde{P}_{2}$ contains only value 0 . For $S$, the next conflict-free " 1 " will occur no later than $l$, where $l$ satisfies

$$
\begin{cases}l \equiv 0, & \bmod L \\ l \equiv k, & \bmod p(2 p-1) \\ 0 \leq l<p(2 p-1) L . & \end{cases}
$$

That is, $l=k L$. By Lemma $6.3, k \leq 2 p$.

The estimate in Theorem 6.2 is relatively crude. It is believed that a smaller bound is possible, maybe with some modification in the sequence design. The CRT sequence approach requires working with relatively prime parameters. It is possible to relax this requirement by using Generalized Prime Sequences, as described in [1].

\section{CONCLUSION}

In this paper, we have proposed a new approach to MAC design that is based on information obtained from a GNSS. The approach merges idea from code-based scheduling and protocol sequence. It allows for differences in time synchronization among different users and enables the possibility of traffic load fine tuning based on geographic locations. The concept of spatial reuse for transmission sequences is believed to be novel and provides a new perspective on addressing MAC problems. This opens up a potentially fertile direction for further investigation. 


\section{REFERENCES}

[1] Y. Wu, K. W. Shum, Q. Su, W. S. Wong, and L. Shen, "Safety message broadcast in vehicular ad hoc networks based on protocol sequences," IEEE Trans. Veh. Technol., Sep. 5, 2013, DOI: 10.1109/TVT.2013. 2279857.

[2] G. Solomon, "Optimal frequency hopping sequences for multiple access," in Proc. Symp. Spread Spectr. Commun., 1973, pp. 33-35.

[3] I. Chlamtac and A. Faragró, "Making transmission schedules immune to topology changes in multi-hop packet radio networks," lEEE/ACM Trans. Netw., vol. 2, no. 1, pp. 23-29, Feb. 1994.

[4] C. H. Rentel and T. Kunz, "Reed-Solomon and Hermitian code-based scheduling protocols for wireless ad hoc networks," in Ad-Hoc, Mobile, and Wireless Networks. Berlin, Germany: Springer-Verlag, 2005, pp. 221-234.

[5] C. H. Rentel and T. Kunz, "Bounds and parameter optimization of medium access control coding for wireless ad hoc and sensor networks," Ad Hoc Netw., vol. 10, no. 1, pp. 128-143, Jan. 2012.

[6] J. L. Massey, "The capacity of the collision channel without feedback," in Proc. IEEE Int. Symp. Inf. Theory, Les Arcs, France, Jun. 1982, p. 101.

[7] J. L. Massey and P. Mathys, "The collision channel without feedback," IEEE Trans. Inf. Theory, vol. IT-31, no. 2, pp. 192-204, Mar. 1985.

[8] E. N. Gilbert, "Cyclically permutable error-correcting codes," IEEE Trans. Inf. Theory, vol. IT-9, no. 3, pp. 175-182, Jul. 1963.

[9] Q. A Nguyen, L. Györfi, and J. L. Massey, "Constructions of binary constant-weight cyclic codes and cyclically permutable codes," IEEE Trans. Inf. Theory, vol. 38, no. 3, pp. 940-949, May 1992.

[10] K. W. Shum and W. S. Wong, "Construction of short protocol sequences with worst-case throughput guarantee," in Proc. IEEE Int. Symp. Inf. Theory, Austin, TX, USA, Jun. 2010, pp. 1828-1832.

[11] K. W. Shum, Y. Zhang, and W. S. Wong, User-Irrepressible Sequences. Berlin, Germany: Springer-Verlag, Sep. 2010, pp. 88-101.

[12] K. W. Shum and W. S. Wong, "Construction and applications of CRT sequences," IEEE Trans. Inf. Theory, vol. 56, no. 11, pp. 5780-5795, Nov. 2010

[13] A. A. Shaar and P. A. Davies, "A survey of one-coincidence sequences for frequency-hopped spread-spectrum systems," Proc. Inst. Elect. Eng. F-Commun., Radar Signal Process., vol. 131, no. 7, pp. 719-724, Dec. 1984

[14] G. Yang and W. C. Kwong, Prime Codes with Applications to CDMA Optical and Wireless Networks. Norwood, MA, Reading: Artech House, 2002.

[15] J. Broch, D. A. Maltz, D. B. Johnson, Y.-C. Hu, and J. Jetcheva, "A performance comparison of multi-hop wireless ad hoc network routing protocols," in Proc. ACM/IEEE Int. Conf. Mobile Comput. Netw., Dallas, TX, USA, Oct. 1998, pp. 85-97.
[16] H. Menouar, F. Filali, and M. Lenardi, "A survey and qualitative analysis of MAC protocols for vehicular ad hoc networks," IEEE Wireless Commun., vol. 13, no. 5, pp. 30-35, Oct. 2006.

[17] W. S. Wong, "New protocol sequences for random access channels without feedback," IEEE Trans. Inf. Theory, vol. 53, no. 6, pp. 2060-2071, Jun. 2007.

[18] K. W. Shum, W. S. Wong, C. W. Sung, and C. S. Chen, "Design and construction of protocol sequences: Shift invariance and user irrepressibility," in Proc. IEEE Int. Symp. Inf. Theory, Seoul, Korea, Jun. 2009, pp. 1368-1372.

[19] K. W. Shum, W. S. Wong, and C. S. Chen, "A general upper bound on the size of constant-weight conflict-avoiding codes," IEEE Trans. Inf. Theory, vol. 56, no. 7, pp. 3265-3276, Jul. 2010.

[20] V. H. MacDonald, "The cellular concept," Bell Syst. Tech. J., vol. 58, no. 1, pp. 15-43, Jan. 1979

[21] J.-H. Ju and V. O. K. Li, "An optimal topology-transparent scheduling method in multi-hop packet radio networks," IEEE/ACM Trans. Netw., vol. 6, no. 3, pp. 298-306, Jun. 1998.

[22] V. R. Syrotiuk, C. J. Colbourn, and A. C. Ling, "Topology-transparent scheduling for MANETs using orthogonal arrays," in Proc. Int. Conf. Mobile Comput. Netw., San Diego, CA, USA, Sep. 2003, pp. 43-49.

[23] S. Basagni and D. Bruschi, "A logarithmic lower bound for TimeSpread Multiple-Access (TSMA) protocols," Wireless Netw., vol. 6, no. 2, pp. 161-163, May 2000.

[24] J. Justesen and T. Høholdt, A Course in Error-Correcting Codes. Zürich, Switzerland: Eur. Math. Soc., 2004.

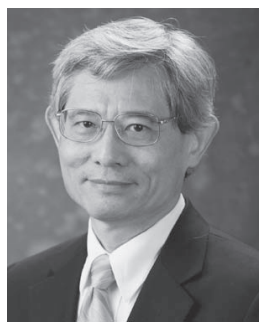

Wing Shing Wong (M'81-SM'90-F'02) received the combined Master's and Bachelor's degree from Yale University, New Haven, CT, USA, and the M.S. and Ph.D. degrees from Harvard University, Cambridge, MA, USA.

He joined AT\&T Bell Laboratories in 1982 and moved to The Chinese University of Hong Kong, Hong Kong, in 1992, where he is currently a Professor of information engineering and the Dean of the Graduate School. From 1995 to 2003, he was the Chairman of the Department of Information Engineering. From 2003 to 2005, he served as a Science Advisor at the Innovation and Technology Commission of the HKSAR government and was the Board Director and Chairman of the Technical Committee of the Hong Kong Applied Science and Technology Research Institute. He has participated in a variety of research and development projects on topics ranging from mobile communication, nonlinear filtering, and information-based control. 